\title{
BMJ open \\ A randomised controlled trial to improve the role of the general practitioner in cancer rehabilitation: effect on patients' satisfaction with their general practitioners
}

To cite: Bergholdt SH, Hansen DG, Larsen PV, et al. A randomised controlled trial to improve the role of the general practitioner in cancer rehabilitation: effect on patients' satisfaction with their general practitioners. BMJ Open 2013;3:e002726. doi:10.1136/bmjopen-2013002726

- Prepublication history and additional material for this paper is available online. To view these files please visit the journal online (http://dx.doi.org/10.1136/ bmjopen-2013-002726).

Received 14 February 2013 Revised 21 May 2013 Accepted 29 May 2013

This final article is available for use under the terms of the Creative Commons Attribution Non-Commercial 3.0 Licence; see http://bmjopen.bmj.com

For numbered affiliations see end of article.

Correspondence to Dr Stinne Holm Bergholdt; sbergholdt@health.sdu.dk

\section{ABSTRACT}

Objective: To test whether a complex intervention facilitating early cancer rehabilitation by involvement of the general practitioner (GP) soon after diagnosis improves patients' satisfaction with their GPs.

Design: A cluster randomised controlled trial. All general practices in Denmark were randomised to an intervention or a control group before the start of the study. Patients included those with cancer who were subsequently allocated to either group based on the randomisation status of their GP.

Participants: Adult patients with cancer treated for incident cancer at the public regional hospital (Vejle Hospital, Denmark) were included between May 2008 and February 2009. A total of 955 patients registered with 323 practices were included, of which 486 patients were allocated to the intervention group and 469 to the control group.

Intervention: The intervention included a patient interview assessing the need for rehabilitation, improved information from the hospital to GPs including information on the patients' current needs along with information about needs of patients with cancer in general. Further, GPs were encouraged to proactively contact the patients and facilitate the patients' rehabilitation course.

Outcome measures: 6 months after inclusion of the patient, patient satisfaction with their GP during the last 12 months in five different dimensions of GP care was assessed using the Danish version of the EuroPEP (European Patients Evaluate General Practice Care) questionnaire (DanPEP). 14 months after inclusion, patient satisfaction with the GP regarding the cancer course and GP's satisfaction with own contribution to the patients' rehabilitation course were assessed using ad hoc questions specifically designed for this study.

Results: No overall effect of the intervention was observed. Subgroup analysis of the patients with breast cancer showed statistically significant improvement of satisfaction with the GP in two of the five DanPEP dimensions.

Conclusions: This complex intervention aiming at improving GPs' services in cancer rehabilitation had no impact on patient satisfaction.

\section{ARTICLE SUMMARY}

Article focus

- The role of general practitioners (GPs) in cancer rehabilitation is not well defined.

- Early involvement of the GP in cancer rehabilitation is supposed to improve patient satisfaction with the GP.

- Improved information from the hospital to GPS about individual patients' rehabilitation needs is supposed to improve patients' satisfaction with their GPs' contribution to their cancer course and the GPs' satisfaction with their own contribution to the patients' rehabilitation course.

Key messages

- Early involvement of the GP including improved information about individual rehabilitation needs did not improve either patient or GP satisfaction with the GP's contribution to the cancer course or patient satisfaction with the GP in general.

- The patient satisfaction with the GP among patients with cancer in the study was low compared to patient satisfaction observed in a mixed patient population.

- There was low agreement between patient and GP satisfaction.

- Subgroup analyses comprising patients with breast cancer indicate that the intervention may be effective for some groups of patients with cancer

Strengths and limitations of this study

- The effects of GPs' involvement in cancer rehabilitation targeting a broad group of patients with cancer were evaluated in a large randomised controlled study.

- Validated outcome measures were used, but other relevant effects may have been overlooked.

Trial registration: ClinicalTrials.gov, registration ID number NCT01021371 


\section{INTRODUCTION}

In the past decade, there has been an increasing focus on the human costs of cancer for the individual patient and their relatives. Patients with cancer experience different courses of disease, needs and unmet needs. They have different resources and motivations for receiving help, and adjusting to and coping with critical situations. This complexity of the consequences of cancer adds to the challenges of organising a comprehensive and individual rehabilitation course that embraces the complex of physical, psychosocial, sexual and financial problems and unmet needs experienced by patients with cancer. ${ }^{1-8}$

The general practitioner (GP) is regarded as a central person in coordinating each patient's rehabilitation course, since they often have prior knowledge about the patient's resources and comorbidity, ${ }^{9-14}$ which are factors that might influence the quality and benefits of the patient's rehabilitation. Also, studies have shown that patients would like their GP to be involved and proactive during the course of disease. ${ }^{2} 1516$ However, the GP is often sidelined during the cancer treatment, which complicates early rehabilitation as well as the critical transition phase from the hospital to the general practice after the end of the treatment. ${ }^{16-18}$ This may contribute to the low patient satisfaction with respect to the GPs' interest in and contribution to the course of the cancer disease observed in previous studies. ${ }^{1} 219$

The active involvement of GPs early after diagnosis may facilitate the important supportive role of GPs during the cancer course and lead to more continuous contacts, thus improving the relationship between patients and GPs in subsequent cancer care. ${ }^{11} 122021$ The purpose of this paper is to evaluate the effects of an intervention encouraging the early involvement of GPs on satisfaction of patients with cancer with their GP in general, and specifically in relation to the cancer course. Furthermore, the GPs' self-reported satisfaction with their own contribution to their patients' physical and psychosocial rehabilitation will be explored.

The present paper is part of a series of papers evaluating the effects of the intervention. We have previously reported that the intervention had no statistically significant effect on the patients' quality of life as the primary outcome $^{22}$ or on the level of psychological distress, ${ }^{22}$ on GPs' proactivity or on the patients' participation in rehabilitation activities ${ }^{23}$ as secondary outcomes. In this light, one might question whether we could reasonably expect any effect on patient satisfaction. However, the intervention was expected to improve the communication between the hospital and GPs as well as between GPs and patients, which in itself may improve patient satisfaction with GPs, even if there were no detectable effects on, for example, the quality of life or psychological distress.

\section{MATERIALS AND METHODS}

We conducted a cluster randomised controlled trial, testing whether a complex intervention facilitating early cancer rehabilitation by involvement of GPs early after diagnosis improves patients' satisfaction with their GPs.

\section{Participants}

All adult patients ( $\geq 18$ years) newly diagnosed with cancer and admitted to Vejle Hospital between 12 May 2008 and 28 February 2009 were assessed for eligibility. Patients were included if treated at Vejle Hospital for a cancer diagnosed within the previous 3 months and if listed with a general practice. Patients with carcinoma in situ or non-melanoma skin cancers were not included (figure 1). Eligible patients were identified across hospital departments, cancer types, cancer stages and potential rehabilitation needs by use of electronic patient files. ${ }^{24}$ Two rehabilitation coordinators (RCs), both nurses with oncological experience, assessed all patients for eligibility and managed the intervention. RCs were assigned exclusively to the project and did not take part in the daily routines at the hospital ward.

\section{Setting}

The study was conducted at Vejle Hospital, a public general hospital in the Region of Southern Denmark (1.2 million inhabitants). Patients with cancer were allocated from all of Denmark.

The Danish publicly funded healthcare system ensures free access to general practice, and GPs function as gatekeepers to the rest of the healthcare system. More than 98\% of all Danish residents are registered with a general practice. ${ }^{25}$ All Danish GPs have electronic patient filing systems and all information from the hospitals, including discharge letters, are received electronically and stored directly in the patient files. On average, a GP encounters nine patients with incident cancer in 1 year. $^{26}$

The GPs' options for referring patients to relevant rehabilitation activities vary between the different municipalities, just as the availability of private patient associations and other relief organisations. These variations might influence the quality of the rehabilitation course between patients in different parts of the country.

\section{Development and piloting of questionnaires and intervention}

Before designing the intervention, we reviewed papers, reports and textbooks about the problems faced by patients with cancer and GPs with respect to individual rehabilitation and continuity across healthcare sectors. $^{1511121721}$

The questionnaires and the procedures of identification, assessment and inclusion of patients were pilot tested prior to the start of the study. The procedures have been described in detail previously. ${ }^{24}$

\section{Intervention}

The intervention comprised a patient interview about rehabilitation needs conducted by an RC, followed by information to the GP about the patient's individual rehabilitation needs and the rehabilitation needs of 
Figure 1 Study flow.

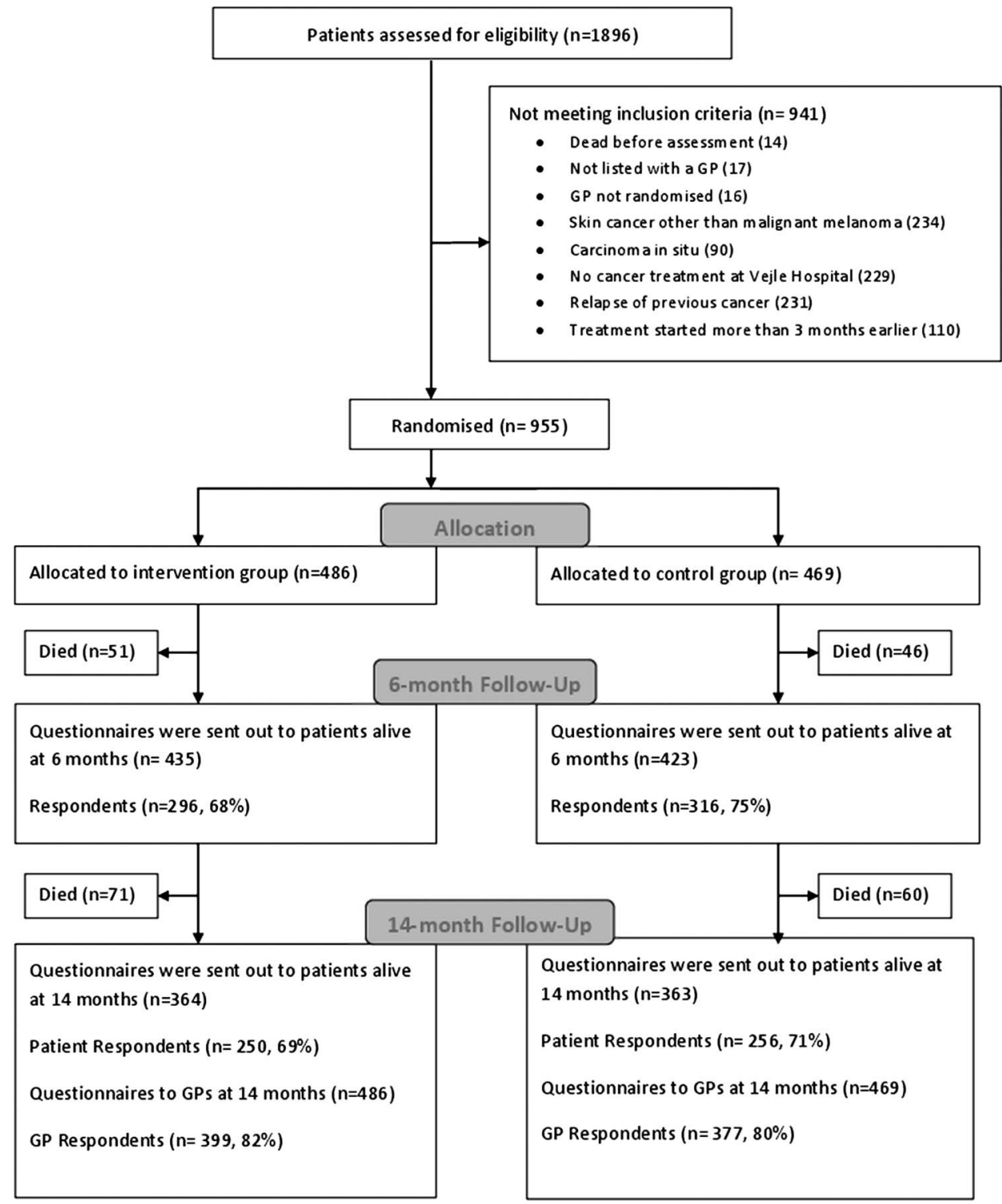

patients with cancer in general (figure 2). This improved information from the hospital to the GP was supplemented by encouraging the GP to proactively contact the patient to facilitate the rehabilitation process.

Patient interviews were conducted according to an interview guide ${ }^{27}$ and based on a list of general needs and problems among patients with cancer (figure 2) constructed on the basis of existing knowledge. ${ }^{1} 4 \quad 5$ Interviews were most often conducted at the hospital, but in some cases by phone. During the interview, the concept of rehabilitation was explained, and the individual needs for physical, psychological, sexual, social, workrelated and finance-related rehabilitation were identified. It was emphasised that physical, psychological, sexual, social, work-related and financial problems might occur at any time and change during the disease trajectory. ${ }^{3} 28$ In order to address these problems, patients were advised to consult their GP during and after treatment.
The patients gave oral consent to their GP being informed about their individual problems and needs.

Following each interview, the patient's GP was informed about the patient's actual problems and need for rehabilitation and the GP was encouraged to be proactive, that is, the GP was encouraged to contact the patient personally to offer support and guidance in order to identify and address the actual and future needs for rehabilitation. Further, the GP received an email summarising the information, supplemented by general information about the needs and problems of patients with cancer (figure 2). The information about the individual patient was personally conveyed by phone, if possible, and always sent electronically along with the general information. Patients and GPs in the control group were not contacted by $\mathrm{RCs}^{24}$ and care and communication took place according to usual procedures. Details about the feasibility of the intervention have been published previously. ${ }^{24}$ 


\begin{tabular}{|c|c|}
\hline Psychol ogical level & $\begin{array}{l}\text { - Fear of death or recurrence } \\
\text { - Guilt feelings about being sick } \\
\text { - Anger at general practitioner or "system" for not having } \\
\text { taken action soon enough } \\
\text { - Troubles adjusting to new self-image } \\
\text { - Feeling of being left in limbo after discharge from the } \\
\text { hospital } \\
\text { - Risk of developing depression } \\
\text { - Reconsiderations about priorities in life and how one } \\
\text { - Sexual problems }\end{array}$ \\
\hline Social level & $\begin{array}{l}\text { - Concems about the well-being of spouse, children and } \\
\text { other relatives } \\
\text { - Changed body im age or sexuality } \\
\text { - Changed position/status in marriage, in family, at work, } \\
\text { etc. } \\
\text { - Concems about possible infertility caused by treatment } \\
\text { - Information about patient associations and similar groups } \\
\text { for patients and relatives }\end{array}$ \\
\hline Physical level & $\begin{array}{l}\text { Physical capacity with regard to daily activities, need for } \\
\text { special facilities, homecare, conversions of the home, etc. } \\
\text { - Need for dietary advice, e.g. to prevent umdue weight loss } \\
\text { - Support in order to accept physical changes and late } \\
\text { complications like tiredness, amputation, infertility, pain, } \\
\text { etc. }\end{array}$ \\
\hline Work-related level & $\begin{array}{l}\text { - Concems about losing one's job } \\
\text { - Concems about having to give up one's form er } \\
\text { responsibilities or change field of work due to reduced } \\
\text { ability to work } \\
\text { - Opportunities for financial support during sick-leave, } \\
\text { flexible job, etc. } \\
\text { - Support to keep in contact with workplace during sick- } \\
\text { leave }\end{array}$ \\
\hline Financial level & $\begin{array}{l}\text { - Social rights like mileage allowances, reimbursement of } \\
\text { assistive technology, etc. } \\
\text { - Concems about a decrease in income and consequences } \\
\text { hereof in relation to housing, spouse, children, etc. } \\
\text { - Conditions regarding pension or incapacity benefit }\end{array}$ \\
\hline
\end{tabular}

Figure 2 General needs and problems among cancer patients.

\section{Outcomes and sampling of data}

Patient questionnaires were administered to patients alive at 6 and 14 months after inclusion, and GP questionnaires were administered 14 months after the patient's inclusion. Non-responders were sent one reminder after 3 weeks. ${ }^{24}$ Data were collected in the same way, irrespective of the allocation status.

Patient data on age, sex and cancer diagnosis were obtained from the electronic patient files at Vejle Hospital.

\section{Patient data}

Patient satisfaction with the GP in general was assessed by use of the Danish version of the EuroPEP questionnaire, ${ }^{29}$ the DanPEP (Danish Patients Evaluating General Practice) questionnaire ${ }^{30}$ administered to patients after 6 months of follow-up. The DanPEP questionnaire comprises 23 items evaluating general practice in five different dimensions: 'the doctor-patient relationship' (6 items), 'the medical care' (5 items), 'the information and support' (4 items), 'the organisation of care' (2 items) and 'the GP's accessibility' (6 items). All items refer to the previous 12 months. Answers are given on a five-point Likert scale ranging from 'poor' to 'excellent', with 'acceptable' as the middle option, and with the further option 'not able to answer/not relevant'.

Patient satisfaction with the GP's support during the cancer course was assessed using one ad hoc question in the 14-month questionnaire: "My general practitioner has offered me sufficient support during my course of disease." Answer categories were 'fully agree', 'agree', 'disagree', 'fully disagree', 'don't know' and 'not relevant'.

\section{GP data}

GPs received one questionnaire for each of their patients with cancer participating in the study. This questionnaire included two items measuring the GP's satisfaction with his or her own contribution to the 
individual patient's rehabilitation course by asking "How do you rate your own contribution to the patient's physical rehabilitation" and "How do you rate your own contribution to the patient's psychosocial rehabilitation." Answer categories were 'very satisfactory', 'satisfactory', 'less satisfactory', 'not satisfactory' and 'don't know'. In order to compare the patient's satisfaction with the GP with the GP's satisfaction with his or her own contribution, a new variable incorporating both dimensions of GP answers was constructed.

\section{Sample size}

This paper is part of a randomised controlled trial, with the primary outcome measure being Health Related Quality Of Life (HRQOL) measured by The European Organization for Research and Treatment of Cancer (EORTC) QLQ-C30 questionnaire. ${ }^{31}$ Hence, based on the primary outcome measure, the sample size was estimated to include 144 patients in each group. Since we did not have any data to estimate the effect of clustering prior to the study, we attempted to include patients in each group from a minimum of 144 practice units, allowing for maximum clustering. ${ }^{22}$ Allowing for maximum clustering concerning HRQOL turned out to be very conservative $(95 \%$ intracluster correlation coefficient 0.000 to 0.103$).{ }^{22}$ It is plausible that the cluster effect is similarly low concerning the outcomes of this study.

\section{Randomisation}

Prior to the start of the study, all 2181 general practices in Denmark were randomly allocated to the intervention $(n=1091)$ or control $(n=1090)$ group by the unique provider number of each practice, using a computerised random number generator. Patients were subsequently allocated according to the randomisation status of their GP. Hence, randomisation was performed at the practice level, meaning that all GPs working in the same practice using the same provider number were allocated to the same group. Consequently, a possible spillover effect between GPs and patients from the same practice was minimised.

\section{Blinding}

The study was not blinded. The list of randomisation was available to RCs during assessment of patient eligibility. Allocation status was obvious during the intervention.

\section{Statistical analysis}

Baseline patient demographical and medical characteristics, and age, sex and practice type distribution among responding GPs were explored using descriptive statistics.

In accordance with previous DanPEP and EuroPEP evaluations, ${ }^{29} 3233$ answers on the 1-5 scale of each of the 23 items of the DanPEP questionnaire were dichotomised into 'top evaluations' (corresponding to scores 45 on the 5-point scale) and 'not top evaluation' (corresponding to scores 1-3). For each of the five dimensions, the overall proportion of top evaluations was calculated. 'Not able to answer/not relevant' answers were treated as missing.

Patient satisfaction with the GP's support during the cancer course was dichotomised into 'satisfied' ('fully agree' and 'agree') and 'not satisfied' ('disagree' and 'fully disagree'). 'Don't know' and 'not relevant' answers were treated as missing.

In the GP questionnaire, the two items measuring GP's satisfaction with own contribution to the patient's physical and psychosocial rehabilitation course, respectively, were dichotomised into 'satisfactory' ('satisfactory' and 'very satisfactory') and 'not satisfactory' ('less satisfactory' and 'not satisfactory'). The two items were combined in a new dichotomous variable defined as 'satisfactory', if both items were classified as 'satisfactory', and defined as 'not satisfactory' if one or both items were classified 'not satisfactory'. 'Don't know' answers were treated as missing.

Agreement between patient and GP satisfaction with the GPs' contribution to the cancer course was analysed by comparison of the dichotomised patient satisfaction variable and the combined GP satisfaction variable using Cohen's $\kappa$ statistic.

To analyse the effect of the intervention on patient satisfaction, that is, group differences of the proportion of top evaluations of each of the five DanPEP dimensions, we used mixed effect logistic regression, accounting for possible cluster effects caused by cluster randomisation. All patient outcomes were adjusted for possible confounding effects of the patients' sex and age as a continuous variable. All analyses were conducted as intention to treat, all tests were two-sided and $\mathrm{p}<0.05$ was considered statistically significant. Crude $\left(\mathrm{OR}^{\text {crude }}\right)$ and adjusted ORs $\left(\mathrm{OR}^{\mathrm{adj}}\right)$ are presented with $95 \%$ CIs. Missing values were regarded as missing at random and no imputations were made. Similarly, subgroup analyses of patient and GP satisfaction were conducted among patients with breast cancer .

Statistical analyses were performed using Stata V.11.0 (StataCorp, College Station, Texas, USA). The randomisation procedure was performed using Stata V.10.0.

\section{RESULTS}

In total, 955 patients from 323 general practices fulfilled the inclusion criteria. Of those included, 486 patients from 164 practices were allocated to the intervention group and 469 patients from 159 practices to the control group (figure 1). Patients in the intervention and control groups showed similar baseline demographic and medical characteristics (table 1). However, owing to a large capacity for treating breast cancer at Vejle Hospital, this group is over-represented, while the group of patients with prostate cancer is under-represented compared to the general distribution of cancer types in the Danish population in 2008, where breast cancer accounted for $13.6 \%$ of the incidents, prostate cancer 
Table 1 Baseline demographic and medical characteristics of all included patients $(n=955)$

\begin{tabular}{|c|c|c|}
\hline & Control group $(n=469)$ & Intervention group $(n=486)$ \\
\hline \multicolumn{3}{|l|}{ Age, years } \\
\hline Mean $(95 \% \mathrm{Cl})$ & 63.6 (62.5 to 64.6$)$ & 63.2 (62.2 to 64.3$)$ \\
\hline Median & 64 & 64 \\
\hline Range & 21 to 98 & 28 to 92 \\
\hline \multicolumn{3}{|l|}{ Sex, n (\%) } \\
\hline Male & $134(28.6)$ & $133(27.4)$ \\
\hline Female & $335(71.4)$ & 353 (72.6) \\
\hline \multicolumn{3}{|l|}{ Cancer type, n (\%) } \\
\hline Cancer of the breast & $206(43.9)$ & $201(41.4)$ \\
\hline Cancer of the lung & 69 (14.7) & $75(15.4)$ \\
\hline Malignant melanoma & $44(9.4)$ & $35(7.2)$ \\
\hline Cancer of the rectum/anus & $33(7.0)$ & $45(9.3)$ \\
\hline Cancer of the colon & $29(6.2)$ & $39(8.0)$ \\
\hline Cancer of the ovaries & $12(2.6)$ & 9 (1.9) \\
\hline Cancer of the bilary system & 7 (1.5) & $8(1.6)$ \\
\hline Cancer of the brain & $6(1.3)$ & $8(1.6)$ \\
\hline Cancer of the prostate & $8(1.7)$ & $3(0.6)$ \\
\hline Cancer of the corpus uteri & $6(1.3)$ & $5(1.0)$ \\
\hline Myelomatosis & $6(1.3)$ & $5(1.0)$ \\
\hline Lymphoma & $3(0.6)$ & $4(0.8)$ \\
\hline Unspecified location & $16(3.4)$ & $16(3.3)$ \\
\hline Other diagnoses & $24(5.1)$ & $33(6.8)$ \\
\hline
\end{tabular}

for $12.8 \%$ and lung cancer for $12 \%$, as the three largest cancer types. The distribution of cancer types at Vejle Hospital naturally implies an overrepresentation of female patients with cancer (an almost even number of men and women were diagnosed with cancer in 2008 at a national level). ${ }^{34}$ Responding GPs in the two groups showed similar demographic characteristics (table 2).

Completion rates of the 23 DanPEP items ranged between $65 \%$ and $67 \%$ (558-577 patients of the 858 alive after 6 months of follow-up). No statistically significant differences between the intervention and control groups were observed in any of the five DanPEP dimensions: 'the doctor-patient relationship', 'the medical care', 'the information and support', 'the organisation of care' and 'the GP's accessibility' (table 3). Overall, the percentages of top evaluations of the five dimensions ranged between
$42.6 \%$ (the GP's accessibility) and $58 \%$ (the doctorpatient relationship). Percentages of top evaluations of the single items ranged between $34.4 \%$ and $70.7 \%$.

The completion rate of the question regarding patients' satisfaction with their GP's support during the cancer course was 64\% (466 patients of 727 alive after 14 months of follow-up). No statistically significant difference was observed between groups with regard to patients' satisfaction with their GP's support during the cancer course (table 4).

Questionnaires about the GP's satisfaction with his or her own contribution to the patient's cancer rehabilitation course were sent to GPs of all 955 included patients. We obtained data from GPs regarding 503 patients $(53 \%$ of all 955 patients included) concerning the physical rehabilitation, and regarding 527 patients $(55 \%$ of all

Table 2 Demographic characteristics of responding general practitioners after 14 months of follow-up. General practitioner (GPs) are represented for each returned questionnaire and may thus be represented more than once

\begin{tabular}{lll} 
& Control group & Intervention group \\
\hline Age (years) & $\mathrm{N}=377$ & $\mathrm{n}=398$ \\
Mean $(95 \% \mathrm{Cl})$ & $53.3(52.5$ to 54.1$)$ & $53.3(52.4$ to 54.1$)$ \\
Median & 55 & 55 \\
Range & $34-69$ & $32-70$ \\
Sex, $\mathrm{n}(\%)$ & $\mathrm{N}=377$ & $\mathrm{n}=399$ \\
Male & $263(69.8)$ & $255(63.9)$ \\
Female & $114(30.2)$ & $144(36.1)$ \\
Practice type, $\mathrm{n}(\%)$ & $\mathrm{N}=376$ & $\mathrm{n}=396$ \\
Single-handed practice & $79(21)$ & $67(17)$ \\
Partnership practice* & $297(79)$ & $329(83)$ \\
\hline${ }^{*}$ Two or more GPs working together. & &
\end{tabular}




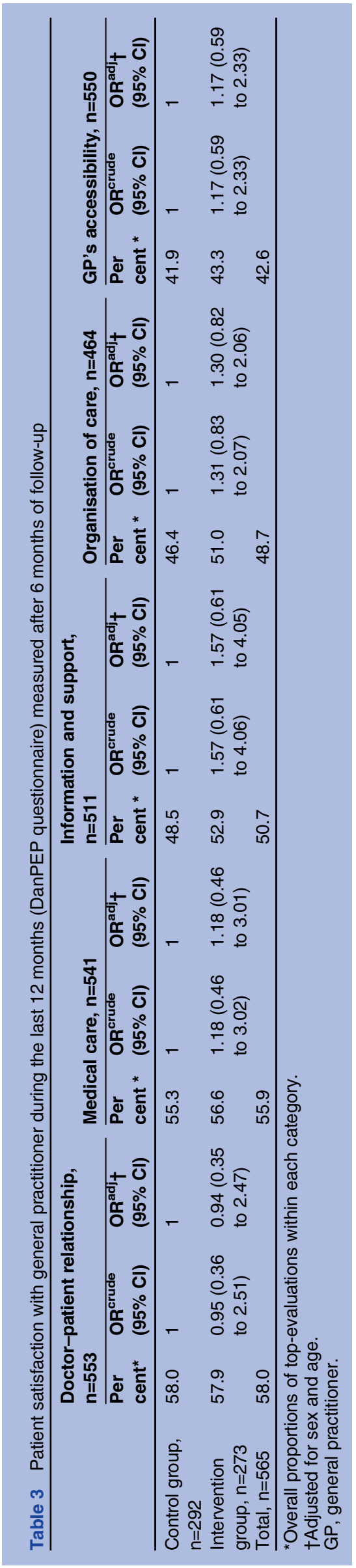

955 patients included) concerning the psychosocial rehabilitation. No statistically significant difference was observed between the intervention and control groups with regard to the GPs' satisfaction with their own contribution to the patients' rehabilitation course (table 5).

The agreement between patients and GPs with regard to satisfaction with the cancer course after 14 months of follow-up was $74 \%$ ( $\kappa 0.19, \mathrm{n}=106$ pairs) in the intervention group and $59 \%(\kappa-0.09, \mathrm{n}=113$ pairs $)$ in the control group.

Subgroup analyses of patients with breast cancer on patient satisfaction with the GP using the DanPEP data at 6 months of follow-up showed a generally higher level of satisfaction with the GP regarding all five dimensions among patients in the intervention group. Regarding the dimensions 'the information and support' $\left(\mathrm{OR}^{\text {adj }}\right.$ $5.51,95 \%$ CI 1.20 to 25.35$)$ and 'the organisation of care' $\left(\mathrm{OR}^{\text {adj }} 2.08,95 \%\right.$ CI 1.03 to 4.21$)$, the differences were statistically significant. Regarding the satisfaction of patients with breast cancer with the GP's contribution to the cancer course at 14 months of follow-up, no difference was observed between groups. Further, no difference was observed in the GPs' satisfaction with their own contribution to the rehabilitation course of patients with breast cancer. Agreement between patients with breast cancer and GPs with regard to satisfaction with the cancer course after 14 months of follow-up was similar to the one observed for the entire patient group.

\section{DISCUSSION \\ Principal findings}

This complex intervention showed no effect on patients' satisfaction with their GP in general or with the GPs' support during the rehabilitation course. Furthermore, no effect of the intervention was observed on the GPs' satisfaction with their own contribution to the patients' rehabilitation course. The agreement between patients' and GPs' satisfaction with the GP's contribution to the cancer course after 14 months of follow-up was low.

\section{Strengths and limitations}

We used the validated DanPEP questionnaire to evaluate patients' general satisfaction with their GPs during the past 12 months. Since this questionnaire was administered to patients after 6 months of follow-up, the level of satisfaction also reflects the diagnostic phase before the intervention was initiated. This may have diluted a possible effect of the intervention on patient satisfaction with the GP.

As there were no validated instruments measuring patient satisfaction with their GP's contribution to their cancer course specifically, we constructed ad hoc questions. Additionally, no validated instruments measuring the GPs' satisfaction with their own contribution to the patient's cancer rehabilitation course were available, and ad hoc questions were constructed. All ad hoc questions were pilot tested and showed acceptable variability and response rates. 
Table 4 Patient satisfaction with the general practitioners' contribution to the rehabilitation course measured after 14 months of follow-up

\begin{tabular}{|c|c|c|c|c|}
\hline & Satisfied, n (\%) & Not satisfied, n (\%) & OR $^{\text {crude }}(95 \% \mathrm{Cl})$ & $\mathrm{OR}^{\mathrm{adj}{ }_{*}}(95 \% \mathrm{Cl})$ \\
\hline Control group, $n=159$ & $105(66.0)$ & $54(34.0)$ & 1 & 1 \\
\hline Intervention group, $n=159$ & $109(68.6)$ & $42(31.4)$ & 1.13 (0.69 to 1.83 ) & 1.14 (0.70 to 1.82$)$ \\
\hline Total, $n=318$ & $214(67.3)$ & $104(32.7)$ & & \\
\hline
\end{tabular}

Cluster randomisation was performed to ensure that GPs working together only handled patients from either the intervention or the control group. Information about the study might have disseminated from GPs in the intervention group to GPs in the control group, but we have no reason to believe that this has caused any significant spillover effect.

The heterogeneity of the study population due to inclusion of patients with various types of cancer, different prognoses, comorbidities and the need for rehabilitation and supportive care may have diluted the effects of the intervention in subgroups of patients with specific diagnoses or problems. Subgroup analyses of satisfaction with the GP among patients with breast cancer using the DanPEP questionnaire showed higher levels of satisfaction with the GP among intervention group patients. Patients with breast cancer are usually younger and socioeconomically advantaged compared to the majority of patients with cancer. This may imply a higher level of psychosocial resources leading to a higher degree of action towards handling of their unmet needs and a greater acceptability towards interventions similar to the one in this study, which may contribute to a higher degree of satisfaction with their GP. We are aware that the choice of a heterogenic sample may have caused some dilution of possible effects of the intervention among subgroups of patients. However, the overall purpose was to test the intervention, which was intended to be relevant to all types of patients with cancer, on patients with cancer in general and thereby to simulate real life in general practice.

After the study was conducted, problems with ceiling effects of the DanPEP questionnaire have been discussed. ${ }^{30}$ However, there is no indication of ceiling effect in our data as the proportion of top evaluations of patients with cancer in our study was considerably lower with regard to all separate items and all five dimensions of care compared to levels in previous DanPEP and EuroPEP surveys. ${ }^{32} 33$

Despite the large sample, this well-conducted, randomised controlled study using validated instruments showed no effect on the patient or GP satisfaction.

\section{Relations to other studies}

Patient satisfaction is widely used as a quality indicator of healthcare services. ${ }^{35}{ }^{36}$ For more than a decade, the EuroPEP questionnaire has been used throughout
Europe to evaluate patient satisfaction with general practice care. ${ }^{29}{ }^{33}$ Evaluations differ between countries, but the overall proportion of top evaluations for all 23 items ranges between $63.9 \%$ and $98.0 \% .^{33}$ A nationwide Danish DanPEP survey conducted in 2002-2005 32 showed proportions of top evaluations similar to the European average. In the Danish study, satisfaction of patients with chronic cancer with their GP was generally higher compared to patients in general and to patients with other chronic diseases. In addition, higher patient satisfaction was found to be strongly associated with increasing age. In our study, patients were considerably less satisfied with their GP with regard to proportions of top evaluations of the 23 items of the DanPEP (ranging between $34.4 \%$ and $70.7 \%$ ) compared to the European and the Danish studies. This may partly be explained by the potential life crisis induced by a diagnosis of cancer, which many patients might have found themselves in at the time of follow-up. Additionally, some patients with cancer are dissatisfied with their GP's handling of their course of diagnosis, which may be reflected as dissatisfaction with the GP in general in the DanPEP data. The increasing adjustment to and coping with the different consequences of the cancer disease over time may explain the higher satisfaction with the GP among patients with chronic cancer. In addition, the threat of a cancer disorder is often said to promote a certain strength and gratefulness for life among the patients surviving it or living with it, which is reflected in higher levels of satisfaction and quality of life than observed among patients in an early phase of their disease, and among people in general.

\section{Meaning}

The complexity of consequences of a cancer disease affecting almost all aspects of the life of patients with cancer demanded a complex intervention embracing as many aspects possible in order to provide an optimal rehabilitation course for each patient. Although no overall effect on patients' or GPs' satisfaction with the GPs' contribution to the cancer course was observed, subgroup analyses of the satisfaction with the GP among patients with breast cancer indicate that this type of intervention may be effective for some groups of patients with cancer. Future research should focus on how to reach other subgroups of patients with cancer with different characteristics and challenges. 


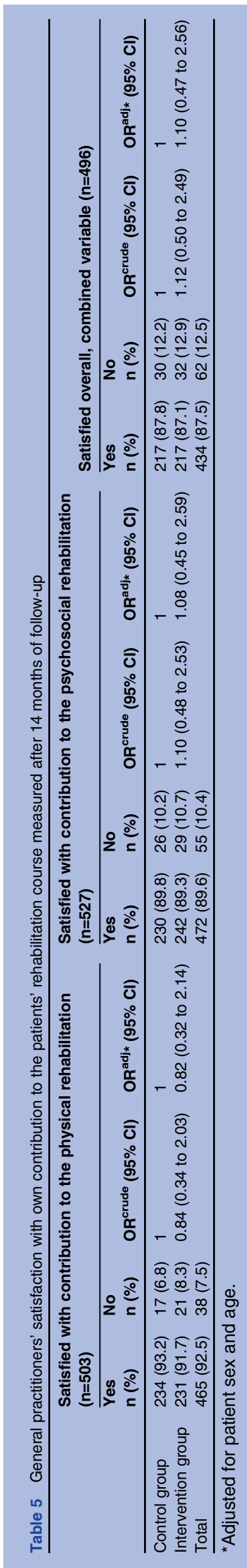

Only $67 \%$ of patients with cancer were satisfied or very satisfied with their GP's contribution to the rehabilitation course. In contrast, close to $90 \%$ of GPs were satisfied or very satisfied with their own contribution to the patients' rehabilitation course. Our results reveal a pronounced lack of agreement between patient and GP satisfaction with the GPs' contribution to the patients' rehabilitation course. The subjective assessment of satisfaction with the support provided may vary between patients and GPs and be influenced not only by many external factors within each group but also by the specific characteristics or experiences of each individual. Future research should focus on the mismatch between GP-experienced and patient-experienced satisfaction with the GPs' contributions, and in particular on how to improve patient satisfaction regarding the GPs' contribution to their rehabilitation course.

The low level of patient satisfaction with regard to all five dimensions of the DanPEP questionnaire of patients in both groups suggests that patients with cancer within the first year after diagnosis may have relatively high expectations to their GP, and require special attention and support from their GP. It would be useful to focus future research on how to best prepare GPs for the complex task of supporting cancer rehabilitation, including how to identify those in need of specific support and proactive care. However, political and financial incentives supporting and defining the GPs' role and activities in cancer rehabilitation are needed in order to provide optimal conditions for GPs to facilitate a successful rehabilitation course for each patient.

In conclusion, this randomised controlled trial showed that a complex intervention aiming at facilitating GPs' role in cancer rehabilitation had no impact on patient satisfaction with their GP or on the GPs' satisfaction with their own contribution to the course of cancer in patients. Low patient satisfaction with the GP, and low agreement between patients' and GPs' satisfaction with the GPs' contribution to the course of cancer in patients were observed.

Author affiliations

${ }^{1}$ Research Unit of General Practice in Odense, National Research Centre for Cancer Rehabilitation, University of Southern Denmark, Odense C, Denmark ${ }^{2}$ Research Unit for General Practice in Copenhagen, Department of Public Health, Faculty of Health Sciences, University of Copenhagen, Copenhagen, Denmark

Acknowledgements The authors wish to thank all patients and healthcare professionals who took part in the study. We also wish to thank Lise Keller Stark, Research Unit of General Practice, University of Southern Denmark, for proofreading the manuscript, and Susanne Døssing Berntsen for managing the datasets.

Contributors SHB, JK, JS and DGH contributed to the conception and study design. SHB, DGH, JK and JS obtained the funding. SHB, JK, JS and DGH wrote the protocol. SHB and DGH were responsible for recruitment of patients. SHB collected and managed the data. PVL was the study statistician. SHB and PVL performed the statistical analysis. SHB, PVL, JK, JS and DGH contributed to the interpretation of data. SHB drafted the first version of the manuscript. SHB, JK, JS, PVL and DGH critically reviewed, revised and supplemented the manuscript. All authors approved the final version. SHB is the guarantor. 
Funding This study was funded by the Danish Cancer Society, the Novo Nordisk Foundation and the Region of Southern Denmark. The National Research Center for Cancer Rehabilitation is funded by the Danish Cancer Society. The authors' work was independent of the funders.

\section{Competing interests None.}

Ethics approval The study was approved by the Danish Data Protection Agency (J.nr. 2008-41-1887). The Regional Committee on Biomedical Research Ethics evaluated the project and concluded that the intervention did not need an approval from the Danish National Committee on Biomedical Research Ethics according to Danish law (Project-ID: S-20082000-7).

Provenance and peer review Not commissioned; externally peer reviewed.

Data sharing statement No additional data are available.

\section{REFERENCES}

1. Groenvold M, Pedersen C, Jensen CR, et al. The cancer patient's world-an investigation of the problems experienced by Danish cancer patients. Copenhagen: Danish Cancer Society, 2006 [in Danish]

2. Mikkelsen T, Sondergaard J, Sokolowski I, et al. Cancer survivors' rehabilitation needs in a primary health care context. Fam Pract 2009;26:221-30.

3. Armes J, Crowe M, Colbourne L, et al. Patients' supportive care needs beyond the end of cancer treatment: a prospective, longitudinal survey. J Clin Oncol 2009;27:6172-9.

4. Hewitt M, Greenfield S, Stovall E. From cancer patient to cancer survivor: lost in transition. Comittee on Cancer Survivorship. Improving Care and Quality of Life, National Cancer Policy Board, Institute of Medicine, and National Research Counsil. Washington DC: The National Academies Press, 2006.

5. Mikkelsen TH, Sondergaard J, Jensen AB, et al. Cancer rehabilitation: psychosocial rehabilitation needs after discharge from hospital? Scand J Prim Health Care 2008;26:216-21.

6. Schmid-Buchi S, Halfens RJ, Dassen T, et al. Psychosocial problems and needs of posttreatment patients with breast cancer and their relatives. Eur J Oncol Nurs 2011;15:260-6.

7. Steele R, Fitch MI. Supportive care needs of women with gynecologic cancer. Cancer Nurs 2008;31:284-91.

8. Fitch MI, Steele R. Supportive care needs of individuals with lung cancer. Can Oncol Nurs J 2010;20:15-22.

9. Olesen F, Dickinson J, Hjortdahl P. General practice-time for a new definition. BMJ 2000;320:354-7.

10. Grunfeld E, Earle CC. The interface between primary and oncology specialty care: treatment through survivorship. J Natl Cancer Inst Monogr 2010;2010:25-30.

11. Kendall M, Boyd K, Campbell C, et al. How do people with cancer wish to be cared for in primary care? Serial discussion groups of patients and carers. Fam Pract 2006;23:644-50.

12. Johansson $\mathrm{B}$, Berglund $\mathrm{G}$, Hoffman $\mathrm{K}$, et al. The role of the general practitioner in cancer care and the effect of an extended information routine. Scand J Prim Health Care 2000;18:143-8.

13. Grunfeld E. Primary care physicians and oncologists are players on the same team. J Clin Oncol 2008;26:2246-7.

14. Bulsara C, Ward AM, Joske D. Patient perceptions of the GP role in cancer management. Aust Fam Physician 2005;34:299-300, 302.

15. Hall S, Gray N, Browne S, et al. A qualitative exploration of the role of primary care in supporting colorectal cancer patients. Support Care Cancer 2012;20:3071-8.

16. Lundstrom LH, Johnsen AT, Ross L, et al. Cross-sectorial cooperation and supportive care in general practice: cancer patients experiences. Fam Pract 2011;28:532-40.

17. Nielsen JD, Palshof T, Mainz J, et al. Randomised controlled trial of a shared care programme for newly referred cancer patients: bridging the gap between general practice and hospital. Qual Saf Health Care 2003;12:263-72.
18. Brazil K, Sussman J, Bainbridge D, et al. Who is responsible? The role of family physicians in the provision of supportive cancer care. $J$ Oncol Pract 2010;6:19-24.

19. Miedema B, MacDonald I, Tatemichi S. Cancer follow-up care. Patients' perspectives. Can Fam Physician 2003;49:890-5.

20. Sisler JJ, Brown JB, Stewart M. Family physicians' roles in cancer care. Survey of patients on a provincial cancer registry. Can Fam Physician 2004;50:889-96.

21. Anvik T, Holtedahl KA, Mikalsen $\mathrm{H}$. When patients have cancer, they stop seeing me-the role of the general practitioner in early follow-up of patients with cancer-a qualitative study. BMC Fam Pract 2006;7:19.

22. Bergholdt SH, Larsen PV, Kragstrup J, et al. Enhanced involvement of general practitioners in cancer rehabilitation: a randomised controlled trial. BMJ open 2012;2:e000764.

23. Bergholdt SH, Søndergaard J, Larsen PV, et al. A randomised controlled trial to improve general practitioners' services in cancer rehabilitation: effects on general practitioners' proactivity and on patients' participation in rehabilitation activities. Acta Oncol 2013:52:400-9.

24. Hansen DG, Bergholdt SH, Holm L, et al. A complex intervention to enhance the involvement of general practitioners in cancer rehabilitation. Protocol for a randomised controlled trial and feasibility study of a multimodal intervention. Acta Oncol 2011; 50:299-306.

25. Pedersen KM, Andersen JS, Sondergaard J. General practice and primary health care in Denmark. J Am Board Fam Med 2012;25: S34-8.

26. PLO Praksistælling. General Practitioners' Organisation Practice Count i. Copenhagen: PLO (General Practitioners' Organisation), 2009. [in Danish].

27. Kurtz SM, Silverman JD. The Calgary-Cambridge Referenced Observation Guides: an aid to defining the curriculum and organizing the teaching in communication training programmes. Med Educ 1996;30:83-9.

28. McDowell ME, Occhipinti S, Ferguson M, et al. Predictors of change in unmet supportive care needs in cancer. Psychooncology 2010;19:508-16.

29. Grol R, Wensing M, Mainz J, et al. Patients in Europe evaluate general practice care: an international comparison. Br J Gen Pract 2000;50:882-7.

30. Vedsted P, Sokolowski I, Heje HN. Data quality and confirmatory factor analysis of the Danish EUROPEP questionnaire on patient evaluation of general practice. Scand J Prim Health Care 2008;26:174-80.

31. Aaronson NK, Ahmedzai S, Bergman B, et al. The European Organization for Research and Treatment of Cancer QLQ-C30: a quality-of-life instrument for use in international clinical trials in oncology. J Natl Cancer Inst 1993;85:365-76.

32. Heje HN, Vedsted P, Sokolowski I, et al. Patient characteristics associated with differences in patients' evaluation of their general practitioner. BMC Health Serv Res 2008;8:178.

33. Petek D, Kunzi B, Kersnik J, et al. Patients' evaluations of European general practice--revisited after 11 years. Int $J$ Qual Health $C$ 2011;23:621-8.

34. Engholm G, Ferlay J, Christensen N, et al. NORDCAN: Cancer Incidence, Mortality, Prevalence and Survival in the Nordic Countries, Version 5.3 (25.04.2013). Association of the Nordic Cancer Registries. Danish Cancer Society. http://www.ancr.nu (accessed 11/05/2013).

35. Lazar EJ, Fleischut P, Regan BK. Quality measurement in healthcare. Annu Rev Med 2013;64:485-96.

36. Heje HN, Gut R, Vedsted P. Patientevaluering af sundhedsvaesenet. [Patient evaluation of health care]. Ugeskr Laeger 2009;171:1666-70. [in Danish].

37. Heje HN, Olesen F, Vedsted P. Patienternes vurdering af de praktiserende laeger. Overordnede resultater fra DanPEP-projektet --del I. [Patients' assessment of their general practitioners--the significance of patient characteristics. General results from the DanPEP_-part I]. Ugeskr Laeger 2010;172:1105-12. [in Danish]. 